\title{
Interstellar Lithium as a probe of the primordial abundance
}

\author{
J. Christopher Howk ${ }^{1}$ \\ ${ }^{1}$ Department of Physics, \\ University of Notre Dame, \\ Notre Dame, IN, USA \\ email: jhowk@nd.edu
}

\begin{abstract}
The cosmic abundance of lithium continues to represent a conundrum, as predictions from BBN theory are inconsistent with measurements in the atmospheres of the lowestmetallicity stars. While there are worries that modifications of the stellar $\mathrm{Li}$ abundances may play a role in this discrepancy, no satisfactory solution has yet been found. We suggest an alternate approach to studying the cosmic abundance of Li: measurements of interstellar gas-phase $\mathrm{Li}$ in low-metallicity environments.
\end{abstract}

Keywords. ISM: abundances, clouds - galaxies: abundances - nuclear reactions, nucleosynthesis, abundances

\section{Introduction}

The primordial Li abundance predicted by standard Big Bang nucleosynthesis (BBN) is a factor of $\gtrsim 2-4$ above the best estimates of the Li abundance in halo star atmospheres (cf., summary in Cyburt et al. 2008). Several possibilities for this discrepancy exist, including destruction of $\mathrm{Li}$ within the stars themselves or the intriguing possibility of new physics (e.g., inhomogeneous nucleosynthesis, Li destruction in the first stars, nonthermal production by particle decays) as discussed elsewhere in these proceedings.

A new approach to estimating the primordial abundance of Li would be extremely useful. Here we reintroduce such an approach, the measurement of interstellar lithium abundances in low-metallicity galaxies. Estimating $\mathrm{Li} / \mathrm{H}$ in the interstellar medium (ISM) of galaxies carries its own potential systematic uncertainties (see Steigman 1996), but they are independent of those that may affect stellar abundance estimates. In addition, the small velocity dispersions of interstellar gas makes it possible to measure the ${ }^{6} \mathrm{Li} /{ }^{7} \mathrm{Li}$ isotopic ratio (Kawanomoto et al. 2009), which can further constrain the origin of Li.

Previous searches for interstellar Li in external galaxies were limited to the SN 1987A sight line in the Large Magellanic Cloud as reported in Vidal-Madjar et al. (1987), ?, and Baade et al. (1991), which had somewhat limited usefulness for placing a limit on the absolute abundance measurements (Steigman 1996). More recently Prodanović \& Fields (2004) suggested it may be possible to measure the gas-phase Li abundances in Galactic high velocity clouds, which are low-metallicity clouds that appear to be falling onto the Galaxy for the first time. While their calculations are overly optimistic (as described below), the rationale for such a measurement is clearly stated. With today's large aperture telescopes, however, it is possible to detect interstellar $\mathrm{Li}$ in other galaxies, and we will report on the first detection of interstellar Li in the Small Magellanic Cloud in an upcoming publication. 


\section{Interstellar lithium abundances}

Measurements of Li in ISM clouds rely on the measurement of neutral lithium in absorption against background light sources using the Li I doublet near $6707 \AA$. However, the direct comparison of the column density of Li I with that of $\mathrm{H}$ I measured in the same way does not directly yield the interstellar $\mathrm{Li}$ abundance, because 1) $\mathrm{Li}^{0}$ is not the dominant ionization stage of $\mathrm{Li}$ in the ISM (that being $\mathrm{Li}^{+}$); and 2) $\mathrm{Li}$ may be incorporated into interstellar dust grains. These effects are such that $\mathrm{Li} \mathrm{I} / \mathrm{H} \mathrm{I} \ll \mathrm{Li} / \mathrm{H}$. Thus, the abundance of $\mathrm{Li}$ in the ISM is given by

$$
\mathrm{Li} / \mathrm{H}=N(\mathrm{Li} \mathrm{I}) N(\mathrm{H})^{-1} x\left(\mathrm{Li}^{0}\right)^{-1} \delta_{\mathrm{Li}}^{-1},
$$

where $N(\mathrm{H}) \equiv N(\mathrm{H} \mathrm{I})+2 N\left(\mathrm{H}_{2}\right)$ is the total hydrogen column density, $x\left(\mathrm{Li}^{0}\right)$ is the ionization fraction of neutral lithium, and $\delta_{\mathrm{Li}}$ is the fraction of all Li present in the gas phase (the "depletion" due to dust). The ionization fraction in this case depends on the density of electrons (and perhaps of dust grains; see ) and the strength of the radiation field.

These corrections can be quite large, with likely values $x\left(\mathrm{Li}^{0}\right)^{-1} \gtrsim 100$ and $\delta_{\mathrm{Li}}^{-1} \sim 4-5$ (Welty et al. 2003). Although the ionization corrections are typically derived in a relative sense using observed ratios of adjacent ions from other elements (e.g., Ca I/Ca II) so that the absolute strength of the radiation field is not important, these can still be quite uncertain. Steigman (1996) has argued that the ratio of Li I/K I can be a better approach to studying the interstellar abundance of $\mathrm{Li}$, given the similar ionization characteristics of these two elements, although one is then in a position of estimating or assuming the $\mathrm{K} / \mathrm{H}$ ratio if one is to estimate $\mathrm{Li} / \mathrm{H}$.

The recent work of Prodanovic \& Fields (2004) did not consider the strong effects of photoionization of $\mathrm{Li} \mathrm{I}$ in their feasibility arguments when discussing measurements in high velocity clouds. The very small amounts of neutral lithium expected for such clouds, coupled with the relatively low column densities of these clouds makes the measurements extremely difficult. It will not be possible to probe $\mathrm{Li}$ in interstellar environments as metal poor as the halo star sample, but by probing gas in a range of metallicities, we will eventually be able to use the interstellar abundances to complement our understanding of the stellar results. Indeed, our first measurement beyond the Milky Way is in the gas of the Small Magellanic Cloud, which has a metallicity $\sim 0.25 Z_{\odot}$. The next generation of very large aperture telescopes should allow us to push this approach to lower metallicity damped Lyman- $\alpha$ systems, although significant uncertainties in the absolute abundances derived through ionization analyses will likely persist.

\section{References}

Baade, D., Cristiani, S., Lanz, T., Malaney, R. A., Sahu, K. S., \& Vladilo, G. 1991, A\&\&A, 251, 253

Cyburt, R. H., Fields, B. D., \& Olive, K. A. 2009, J. Cosmology \& Astroparticle Physics, 11, 012

Kawanomoto, S. et al. 2009, ApJ, 701, 1506

Prodanović, T. \& Fields, B. D. 2004, ApJ (Letters), 616, L115

Steigman, G. 1996, ApJ, 457, 737

Vidal-Madjar, A., Andreani, P., Cristiani, S., Ferlet, R., Lanz, T., \& Vladilo, G. 1987, A\&̋A, 177, L17

Welty, D. E., Hobbs, L. M., \& Morton, D. C. 2003, ApJS, 147, 61 